\title{
Enhanced Proton Acceleration by Laser-Driven Collisionless Shock in the Near-Critical Density Target Embedding with Solid Nanolayers
}

\author{
Yue Chao $\mathbb{D}^{1},{ }^{1}$ Xinxin Yan $\mathbb{D}^{1},{ }^{1}$ Rui Xie $\mathbb{D}^{1},{ }^{1}$ Lihua Cao $\mathbb{D}^{\mathbb{2}, 3}$ Chunyang Zheng, ${ }^{2,3}$ \\ Zhanjun Liu, ${ }^{2,3}$ and Xiantu $\mathrm{He}^{2,3}$ \\ ${ }^{1}$ Center for Applied Physics and Technology, HEDPS, School of Physics and College of Engineering, Peking University, \\ Beijing 100871, China \\ ${ }^{2}$ Institute of Applied Physics and Computational Mathematics, Beijing 100094, China \\ ${ }^{3}$ Center for Applied Physics and Technology, HEDPS and College of Engineering, Peking University, Beijing 100871, China
}

Correspondence should be addressed to Yue Chao; litterel@pku.edu.cn and Lihua Cao; cao_lihua@iapcm.ac.cn

Received 23 April 2021; Revised 5 June 2021; Accepted 21 June 2021; Published 25 June 2021

Academic Editor: Dimitri Batani

Copyright (c) 2021 Yue Chao et al. This is an open access article distributed under the Creative Commons Attribution License, which permits unrestricted use, distribution, and reproduction in any medium, provided the original work is properly cited.

Effects of solid nanolayers embedded in a near-critical density plasma on the laser-driven collisionless shock acceleration are investigated by using two-dimensional particle-in-cell simulations. Due to the interaction of nanolayers and the incident laser, an additional number of hot electrons are generated and an inhomogeneous magnetic field is induced. As a result, the collisionless shock is reinforced within the nanolayer gaps compared to the target without the structured nanolayers. When the laser intensity is $9.8 \times 10^{19} \mathrm{~W} / \mathrm{cm}^{2}$, the amplitude of the electrostatic field is increased by $30 \%$ and the shock velocity is increased from $0.079 \mathrm{c}$ to $0.091 \mathrm{c}$, leading to an enhancement of the peak energy and the cutoff energy of accelerated protons, from $6.9 \mathrm{MeV}$ to $9.1 \mathrm{MeV}$ and 12.2 MeV to $20.0 \mathrm{MeV}$, respectively. Furthermore, the effects of the width of the nanolayer gaps are studied, by adjusting the gap width of nanolayers, and optimal nanolayer setups for collisionless shock acceleration can be acquired.

\section{Introduction}

Ion acceleration via laser-plasma interaction [1] has attracted extensive attention with the rapid development of ultrashort, ultraintense laser technology in recent decades. For its unique characteristics such as high energy and small divergence [2], it has potential scientific applications in a variety of aspects such as inertial confinement fusion (ICF) [3-6], tumor therapy [7, 8], and proton imaging $[9,10]$. Several mechanisms for laserdriven ion acceleration have been proposed and observed experimentally over the past decades, including target normal sheath acceleration (TNSA) [11, 12], radiation pressure acceleration (RPA) [13-15], break-out afterburner (BOA) [16, 17], magnetic vortex acceleration (MVA) [18, 19], and collisionless shock acceleration (CSA) [20-22]. Among them, the RPA and CSA mechanisms have the potential in generating high-energy and quasimonoenergetic ion beams. However, because of the extreme laser conditions required such as ultrahigh intensity and ultrahigh contrast, the evidence for RPA is scant in experiments. Comparatively, CSA is easier to perform and has been reported recently in experiments [23, 24]. In laser-driven CSA, the laser pulse launches a collisionless electrostatic shock in the plasmas by radiation pressure. As the shock propagates forward, it reflects a portion of the background ions to approximately twice the shock velocity due to the strong electrostatic field associated with the shock front. For ions to be reflected, their initial velocity in the shock frame should satisfy [25] $1 / 2 m_{i} u^{2}<e \phi$, where $\phi$ is the potential corresponding to the electrostatic field in the shock front and $m_{i}$ is the mass of the ion. Thus, the energy of reflected ions is strongly dependent on the shock strength. To improve the quality of the CSA, Zhang et al. [26] have proposed to externally apply a strong transverse magnetic field (about $2 \sim 10 \mathrm{kT}$ ) to the plasmas. This field influences the transportation of hot electrons by inhibiting their 
expansion in the longitudinal direction. As more hot electrons are confined near the shock front region, the shock strength is enhanced due to the increased thermal pressure. Therefore, the energy of accelerated ions is greatly enhanced. However, a uniform and stable external magnetic field of such a high intensity is not easy to generate in experiments. Recent works have shown that the nanostructured targets are capable of generating magnetic fields of over $10 \mathrm{kT}$ in both simulations and experiments [27-29]. Besides, the nanostructured targets are much more efficient in laser absorption and hot electron generation compared with planar targets [30-32]. As more hot electrons are generated and collimated inside the gaps of the nanostructured targets, it has the potential advantages for the subsequent ion acceleration. For example, enhanced target normal sheath acceleration in nanostructured targets has been reported to greatly enhance the quality of the ion beam in both simulations [33-35] and experiments [36,37]. At the same time, with the development of surface microtechnology, many novel targets with nanostructures are realized in experiments, such as subwavelength gratings [38], metal nanobrushes [39, 40], and silicon and oxide nanowires [41, 42]. With these technological advances, even more complex nanostructured targets could be feasible in the future.

In this study, we investigate the scheme for enhancing CSA with a solid nanolayered target embedding in the nearcritical plasmas. The fabrication of such a target in the experiment could possibly be realized by inserting the nanolayers in a porous foam target [43]. An intense laser drives the near-critical plasma filled in the gaps of the nanolayers to form a stable collisionless shock as in the regular CSA. Due to the nanolayers, electrons of high density within the mare are heated and dragged out by lasers, giving a contribution to the generation of more hot electrons. Also, the current generated along the surface of the nanolayers induces a strong transverse magnetic field in the near-critical plasma in the gaps. This field acts like a stable external field, inhibiting the longitudinal propagation of hot electrons and focusing them near the shock front. Both effects contribute to the strength of the collisionless shock, leading to an enhancement of the CSA. The effects of the nanolayers on the CSA are studied via two-dimensional particle-in-cell (PIC) simulations, and cases of near-critical plasmas with or without nanolayers are carried out and compared to demonstrate the enhancement of CSA. Simulation results verify the enhancement of proton acceleration, and both the peak energy and cutoff energy for accelerated protons are increased in the case with nanolayers.

This paper is organized as follows. In Section 2, the target setup and simulation parameters are presented. In Section 3, the dynamics and enhancement of CSA are analyzed in detail. In addition, the effect of nanolayers width on CSA is discussed. Section 4 is the conclusion and summary.

\section{Simulation Setup}

To study the effect of the nanolayered target (NLT) on CSA, two-dimensional simulations are carried out with 2D3V PIC codes EPOCH [44]. In the simulation, a circularly polarized laser pulse with wavelength $\lambda_{0}=1 \mu \mathrm{m}$ propagating in the $x$ - direction from the left boundary of the simulation box is incident normally on the target [24]. Its transverse profile is Gaussian, i.e., $I(r)=I_{0} \exp \left(-r^{2} / r_{0}^{2}\right)$, with a focal spot radius of $r_{0}=15 \mu \mathrm{m}$ and a peak intensity $I_{0}=9.8 \times 10^{19} \mathrm{~W} / \mathrm{cm}^{2}$, corresponding to a normalized amplitude $a_{0}=6$ for the circularly polarized laser. The temporal profile is flat-topped with duration $\tau=150 T_{0}$, where $T_{0}=3.3 f s$ is the laser period. For simplicity of analyzing, the NLT is modeled by an array of thin plasma layers with spacing embedded in the homogeneous near-critical $\mathrm{H}^{+}$plasma with a density of $8 n_{c r}$, where $n_{c r}=1.1 \times 10^{21} \mathrm{~cm}^{-3}$ is the critical density. The nanolayers are assumed to be preionized $\mathrm{Au}^{50+}$ plasma with an electron density of $50 n_{c r}$, and they are set immobile in the simulations. Considering the conversion efficiency from laser to hot electrons, a subwavelength nanolayered target is used [45]. Its dimensions are as follows: layer width $d_{1}=0.4 \mu \mathrm{m}$, length $l_{1}=30 \mu \mathrm{m}$, and the distance between the centers of two neighboring layers $d_{2}=5 \mu \mathrm{m}$. The size of the simulation box is $l_{x} \times l_{y}=40 \mu \mathrm{m} \times 30 \mu \mathrm{m}$ with $2000 \times 1500$ grid cells and a time step $\tau=0.01 T_{0}$, and each cell contains 40 particles. Periodic boundary conditions are used in the transverse direction and the nanolayered target is placed in the region of $5 \mu \mathrm{m}<x<35 \mu \mathrm{m}$. Considering that there might be anomalous fields near the boundary, we only take the region of $-10 \mu \mathrm{m}<y<10 \mu \mathrm{m}$ into analysis. For comparison, the case of a planar target (PT) without nanolayers is also considered, where the same laser and plasma parameters are used. To illustrate, the configurations of both targets are sketched in Figure 1. It is worth noticing that our scheme is different from the scheme proposed by $\mathrm{He}$ et al. [46], in which the near-critical plasmas are confined by a thick $\mathrm{Au}^{50+}$ tube. The tube confines the transverse expansion of the hot electrons via the induced electric field. However, in our cases, the incident laser has a large focal spot radius and the transverse expansion is negligible.

\section{Enhancement of the Laser-Driven CSA}

In the laser-driven CSA regime, due to the strong radiation pressure of the incident laser exerted on plasmas, electrons and ions are piled up through the hole-boring effect, forming a density spike moving forward along the longitudinal direction. The laser pulse acts like a piston, pushing the disturbed plasma spike to flow into the undisturbed upstream region. The hole-boring velocity can be estimated [47] as $v_{H B}=\left(I / m_{i} n_{i} c^{3}\right)^{1 / 2}$, where $c$ is the light speed in vacuum, $n_{i}$ is the density of the ion, $I$ is the intensity of the incident laser, and $m_{i}$ is the mass of the ion.

For the parameters in our cases, the hole-boring velocity is $v_{H B} \approx 0.05 \mathrm{c}$. Figure 2 shows the proton density distributions at a different time for the PT (Figures 2(a) and 2(b)) and the NLT (Figures 2(c) and 2(d)). Density spikes of over $30 n_{c r}$ can be observed in both cases and they are drifting to the lower density upstream region. The density ratio of disturbed downstream density spike to the undisturbed upstream plasmas can be obtained from Figure 2, being approximately $\eta=\left(n_{\text {down }} / n_{\text {up }}\right) \approx\left(30 n_{c r} / 8 n_{c r}\right)=3.8$. Furthermore, a significant number of hot electrons are generated to heat the plasma volume by the laser pulse. Figure 3(a) 


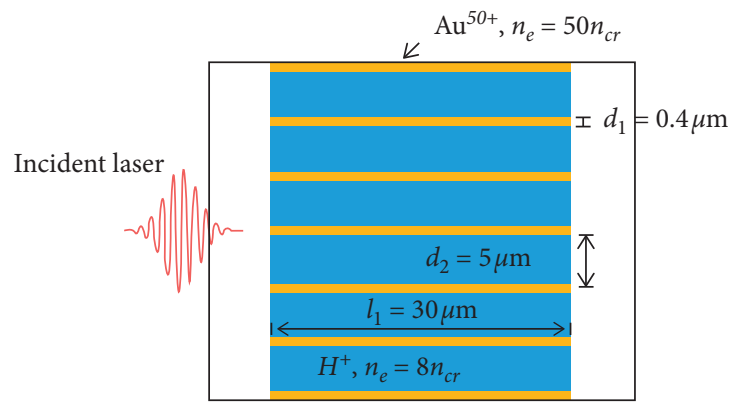

(a)

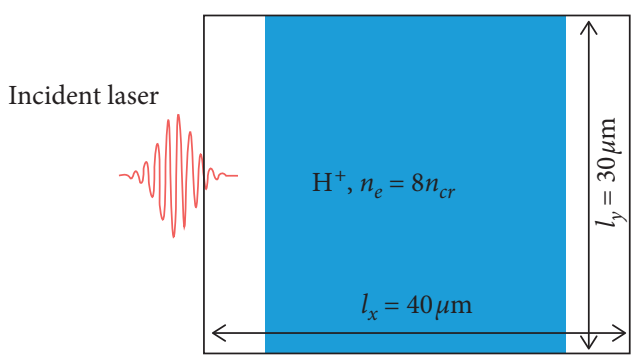

(b)

Figure 1: Target configurations of (a) the NLT and (b) the PT. The NLT consists of near-critical hydrogen plasmas and embedding preionized $\mathrm{Au}^{50+}$ nanolayers.

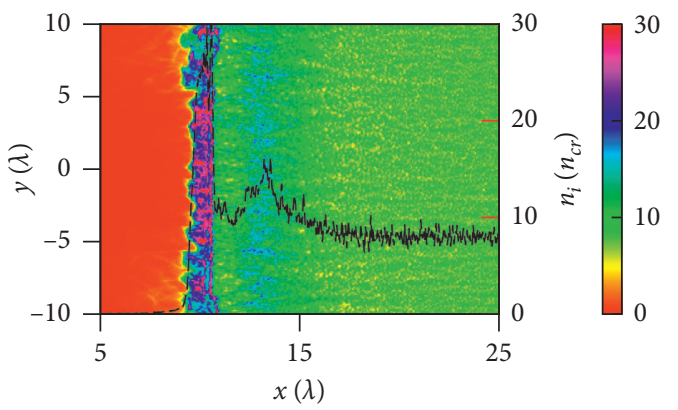

(a)

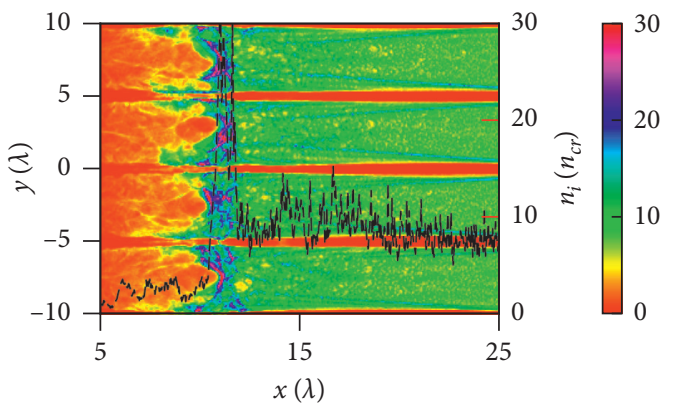

(c)

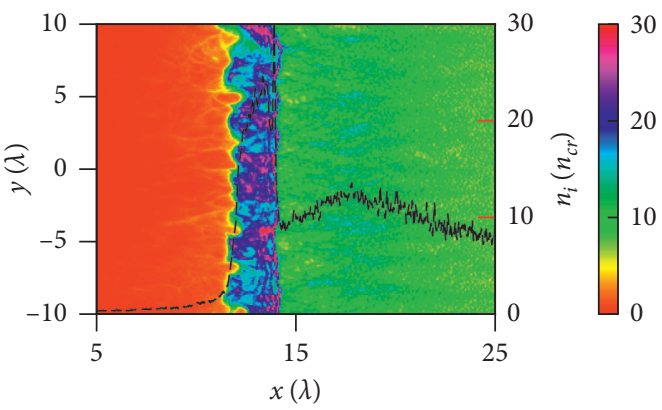

(b)

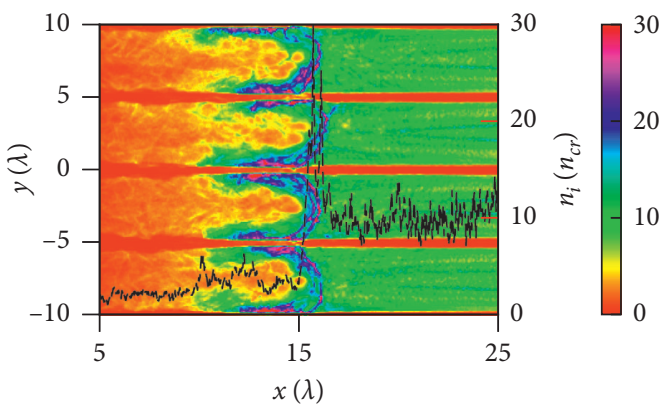

(d)

FIGURE 2: Snapshots and longitudinal density profiles (black dashed lines) of the proton densities at $t=100 T_{0}$ for (a) the PT and (c) the NLT and at $t=150 T_{0}$ for (b) the PT and (d) the NLT. The density peak is approximately $30 n_{c r}$ and the density of undisturbed plasmas in the upstream region is $8 n_{c r}$.

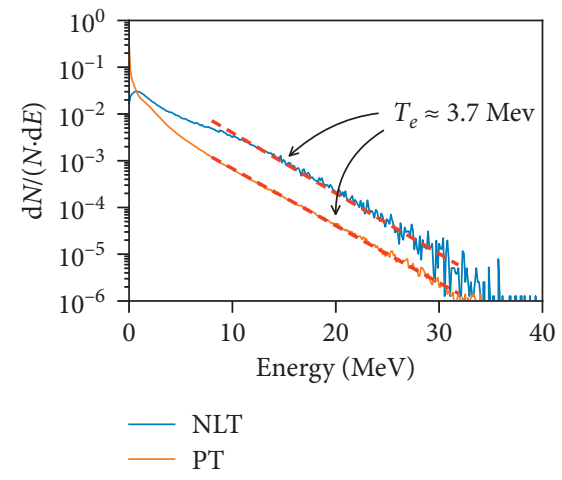

(a)



(b)

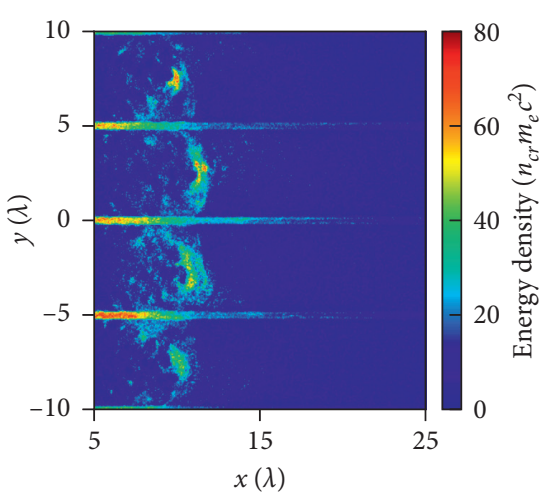

(c)

Figure 3: (a) Energy spectrum of electrons at $t=100 T_{0}$ for the PT (red line) and the NLT (blue line). The hot electron number for the PT is more than that for the NLT, but the temperature for both cases is almost the same as $T_{e} \approx 3.7 \mathrm{MeV}$. Energy densities of the two different electrons from the near-critical plasmas (b) and from the nanolayers (c) at $t=100 T_{0}$. 
presents the electron energy spectrum at $t=100 T_{0}$. There are hot electrons ranging from $10 \mathrm{MeV}$ to $30 \mathrm{MeV}$ and more hot electrons are generated in the NLT due to the nanolayers. By fitting the curves with Maxwellian distribution, the slope of the fitted line gives the hot electron temperature $T_{\text {hot }} \approx 3.7 \mathrm{MeV}$ in both cases, and ion acoustic speed is estimated as $c_{s}=\sqrt{k_{B} T_{e} / m_{i}} \approx 0.06 c$. Figures $3(\mathrm{~b})$ and $3(\mathrm{c})$ show the hot electrons from the near-critical plasmas and the nanolayers, respectively. It can be seen that electrons from the nanolayers also contribute to the hot electron generation near the shock front. In Figure 4, the snapshots of longitudinal momentum phase space of protons at $t=100 T_{0} \quad$ (Figures 4(a) and 4(b)) and $t=150 T_{0}$ (Figures $4(\mathrm{c})$ and $4(\mathrm{~d})$ ) are presented. The phase space distributions show a significant structure of a typical collisionless shock [48]. As labeled in Figure 4(a), the shock velocity is estimated as $v_{s h}=0.08 c$, with a corresponding Mach number $M_{s h} \approx 1.4$, which is approximately the critical Mach number for ion reflection [25] $M_{c r} \approx 1.4$. There are a bunch of reflected protons in the upstream region, and their velocity is approximately twice the shock velocity. Both cases at different times show similar phase space structures and reflected protons. It is worth noticing that the shock velocity and the momentum of reflected protons are significantly increased in the NLT.

To obtain the precise shock velocity, the time evolution of the longitudinal electrostatic field is presented in Figure 5 for the PT (Figure 5(a)) and the NLT (Figure 5(b)), respectively. It can be observed that collisionless shocks are launched at approximately $t=50 T_{0}$ in both cases. The black dashed and dotted lines mark the positions of the peak value. The shock propagates forward at a stable speed after its formation. The reciprocal of the slope of the line gives the velocity of the shock. For the PT, the shock velocity can be estimated from Figure 5(a) as $v_{s h}=0.079 c$, while for the NLT in Figure 5(b), the shock velocity is $v_{s h}=0.091 c$, as much as $25 \%$ higher compared to the former. It is worth noticing that, in both cases, as shown in Figures 5(a) and 5(b), there is an obvious turn of the electrostatic field peak at approximately $50 T_{0}$, marking the formation of the shock, because at the early stage, hole-boring effects dominate and the laser pushes the whole plasma forward. As a result, plasma is piled up and drifts at hole-boring velocity. Once the shock launches, it has a velocity larger than the holeboring velocity, which can be clearly seen from the slope of the black and green lines. At $t<50 T_{0}$ region, the holeboring velocity is estimated to be $v_{H B} \approx 0.06 c$, approximately the value obtained by the theory. To better illustrate the difference between the two cases, the black dashed line from Figure 5(a) is translated to the same position in Figure 5(b), showing a clear divergence of shock velocity between the PT and the NLT. Figure 5(c) shows the energy spectrum of protons accelerated by the collisionless shock wave in the upstream region at $t=150 T_{0}$. Both PT and NLT feature a quasimonoenergetic energy spectrum. In addition, the peak energy and cutoff energy of accelerated protons are both enhanced in the NLT compared to the PT. The peak energy increases from $6.9 \mathrm{MeV}$ to $9.1 \mathrm{MeV}$ by $30 \%$, and the cutoff energy increases as much as $60 \%$ from $12.2 \mathrm{MeV}$ to
20.0 MeV. The increase of shock velocity and proton energy indicates a much stronger shock in the NLT.

The enhancement of the shock strength can be explained by the generation and transportation of hot electrons from nanolayers. As can be seen from the slope of the dashed lines in Figure 3, the temperature of hot electrons is almost the same in both cases. But with solid nanolayers, the number of hot electrons is increased due to the laser-nanolayer interaction. Besides, the current in the nanolayers generates a strong and inhomogeneous transverse magnetic field $B_{z}$, as shown in Figures 6(a) and 6(b), for the PT and the NLT, respectively, where $B_{z}$ is normalized by $m_{e} \omega_{0} / e$. The induced $B_{z}$ inhibits the propagation of the hot electrons in the $x$ direction. Furthermore, due to the nonhomogeneity of $B_{z}$ in the $y$-direction, it exerts a magnetic pressure of $\nabla B^{2} / 8 \pi$ on the hot electrons, expelling them from the region near the nanolayers and pushing them towards the center of the gap, leading to a further accumulation of the hot electrons. The accumulation of hot electrons results in an increased thermal pressure in the shock front region, which can be expressed as $P_{e} \approx n_{h} T_{h}$, where $n_{h}$ and $T_{h}$ are the density and temperature of hot electrons, respectively. This thermal pressure leads to the enhancement of shock strength by increasing the electric field $E_{x} \propto \nabla P_{e}$, where $E_{x}$ is the electric field associated with the shock front. To illustrate, Figures 6(c) and 6(d) show the energy density distribution of hot electrons. It can be seen that electrons are focused and more energetic in the NLT. However, in the PT, they are scattered and distributed in a wider region, leading to a weaker shock. To further illustrate, $E_{x}$ associated with the shock front at $t=100 T_{0}$ and $t=$ $150 T_{0}$ is exhibited in Figures $7(\mathrm{a}), 7(\mathrm{~b}), 7(\mathrm{~d})$, and $7(\mathrm{e})$, normalized by $m_{e} \omega_{0} c / e$. Figures $7(\mathrm{c})$ and $7(\mathrm{f})$ compare $E_{x}$ by averaging them over the $y$-direction. We can see that the peak value of $E_{x}$ is increased as much as $30 \%$ compared to that in the PT at different moments and that the shock in the NLT propagates faster than that in the PT, indicating a stronger shock.

Furthermore, nanolayered targets with different gap widths are investigated to find out a possibly optimal parameter. NLT with corresponding $d_{2}=3,5,7,10 \mu \mathrm{m}$ and PT are separately applied in the simulations for comparison. Figure 8 shows the energy spectra of accelerated protons in different cases. As we can see, in all cases of NLT, the peak energy and cutoff energy are increased compared to the PT. As the gap width decreases from $10 \mu \mathrm{m}$ to $5 \mu \mathrm{m}$, both the peak energy and cutoff energy increase, while the energy spectra of the protons broaden and become quasimonoenergetic. However, as the gap width further decreases to $3 \mu \mathrm{m}$, the peak energy remains the same. But the cutoff energy and the proportion of protons with high energy decreases, meaning decreased conversion efficiency from laser to accelerated protons. While the nanolayers contribute to the hot electron generation and induce the magnetic field, they can also reflect the incident laser. As the gap width decreases, more nanolayers interact with the incident laser, and the proportion of reflected laser will increase. Besides, the hot electrons not only enhance the shock strength but also heat the plasmas in the upstream region. When the gap width decreases, more hot electrons are generated, meaning 


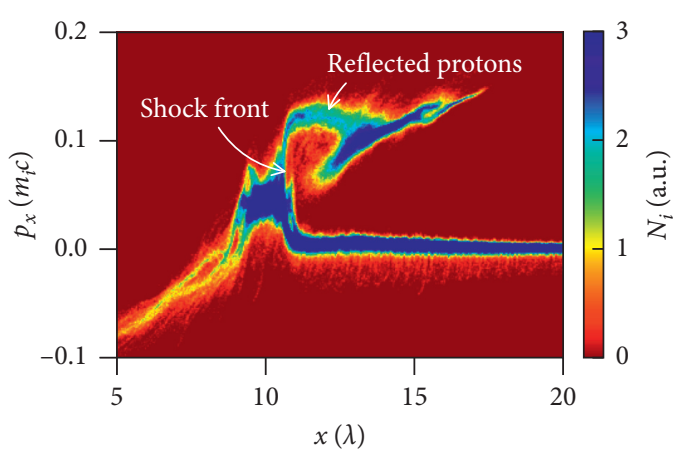

(a)

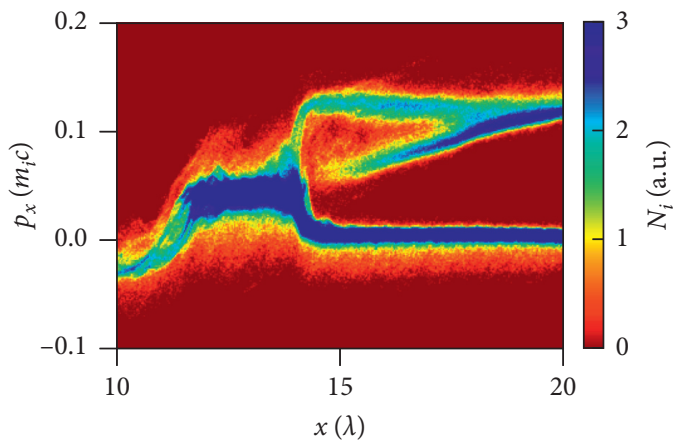

(c)



(b)

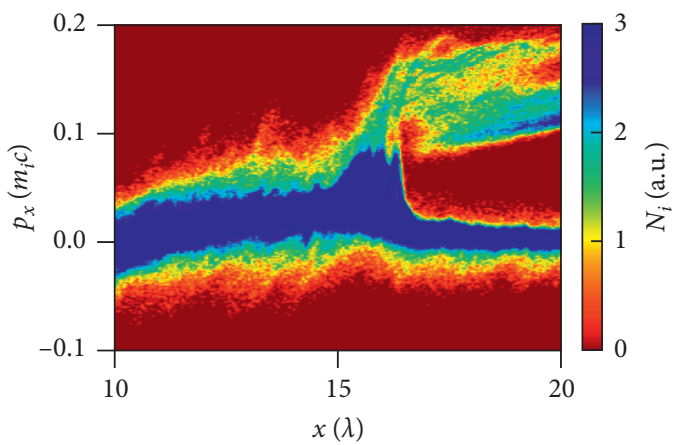

(d)

Figure 4: Phase space $\left(p_{x}-x\right)$ of protons are plotted for the PT (a) and the NLT (b) at $t=100 T_{0}$ and for the PT (c) and the NLT (d) at $t=150 T_{0}$.

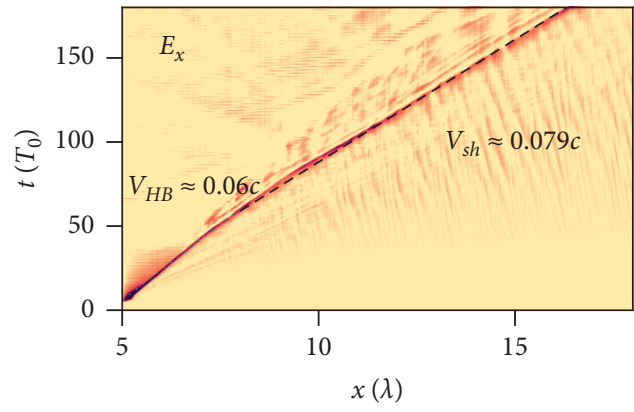

(a)

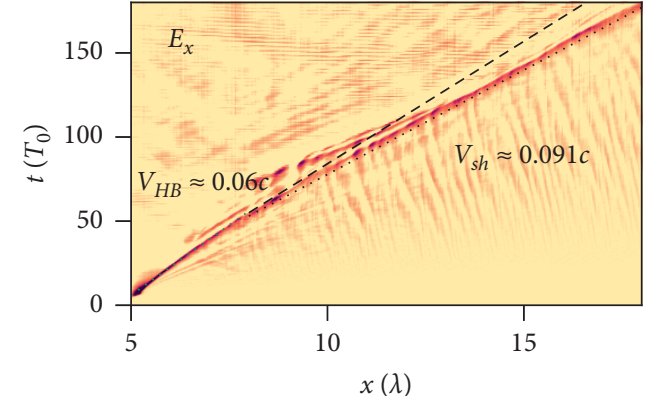

(b)

Figure 5: Continued. 


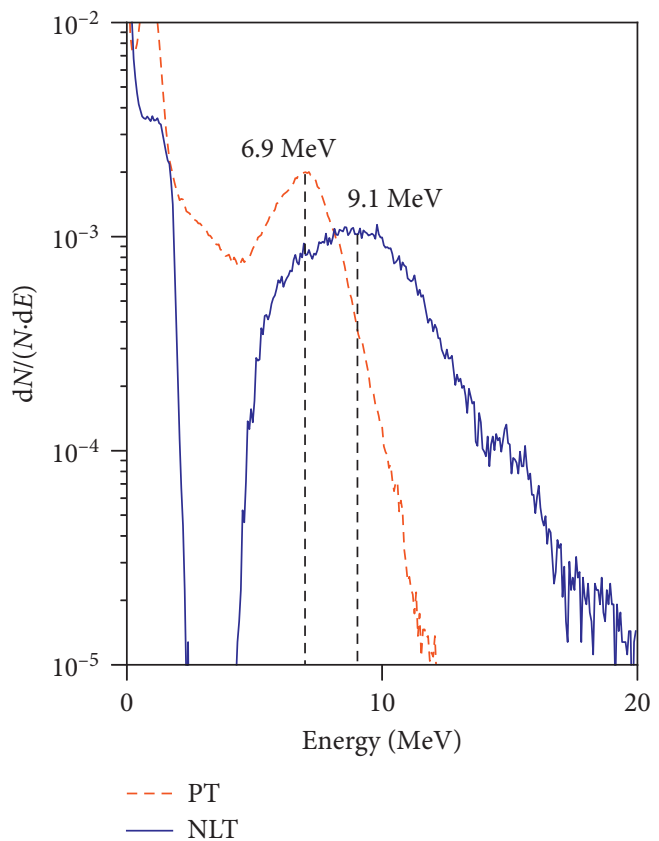

(c)

Figure 5: Time evolution of longitudinal electrostatic field for (a) the PT and (b) the NLT, where the black dashed line marks the shock front for the PT and the dotted line for the NLT. The hole-boring mechanism in the early stage is also marked as green dashed line, and the holeboring velocity $v_{H B} \approx 0.06 \mathrm{c}$. (c) Energy spectrum of accelerated protons in the upstream region at $t=150 T_{0}$.

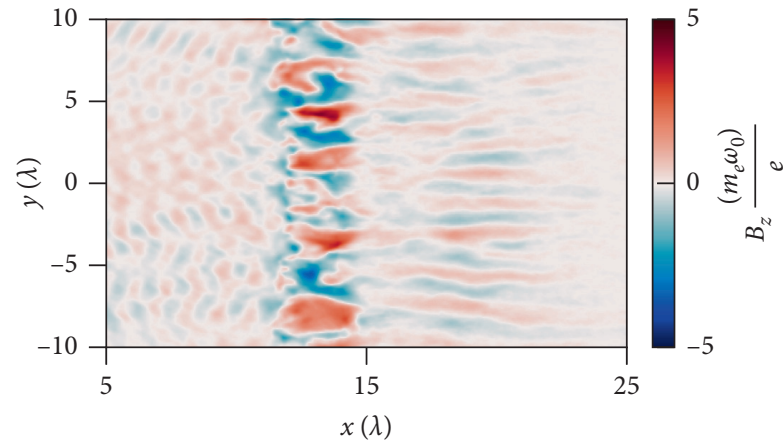

(a)

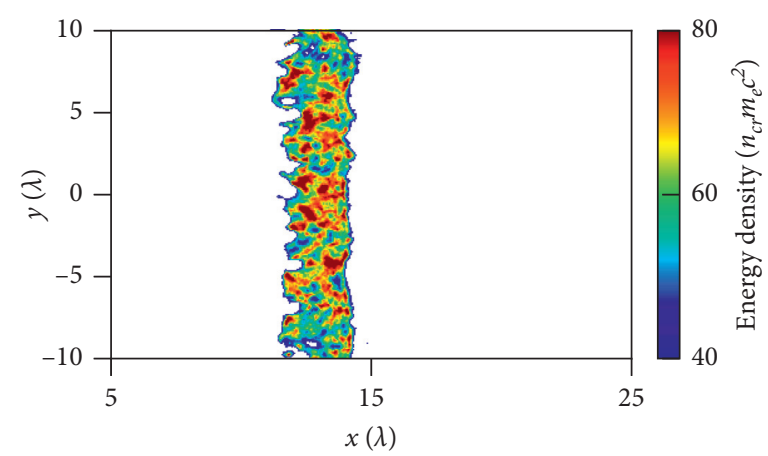

(c)

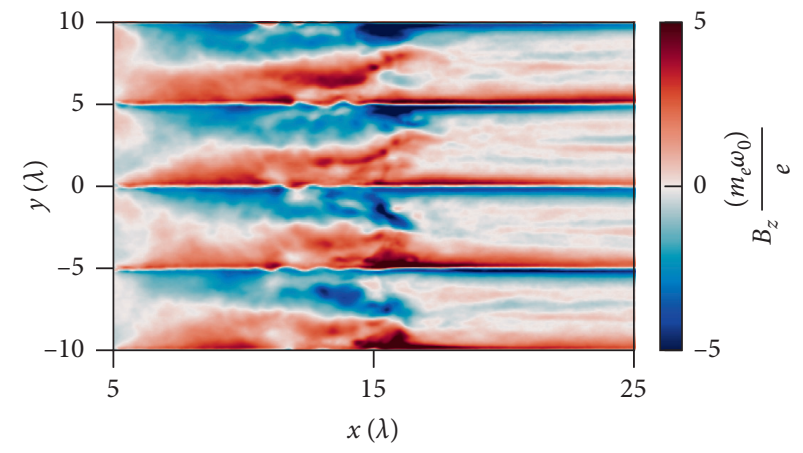

(b)

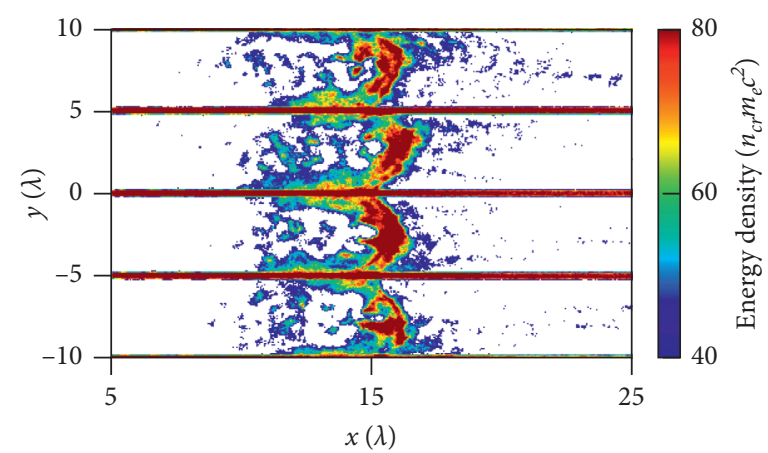

(d)

Figure 6: The transverse magnetic fields $B_{z}$ and the energy density distributions of hot electrons at $t=150 T_{0}$ for $(\mathrm{a}, \mathrm{c})$ the PT and (b, d) the NLT. $B_{z}$ is normalized by $m_{e} \omega_{0} / e$, and the energy density is normalized by $n_{c r} m_{e} c^{2}$. The average magnetic field near the shock front is about $10 \mathrm{kT}$. These hot electrons have a typical gyro-radius of $r_{e} \approx v_{T e}\left(m_{e} c^{2} / e B_{z}\right) \approx 0.5 \mu \mathrm{m}$. 


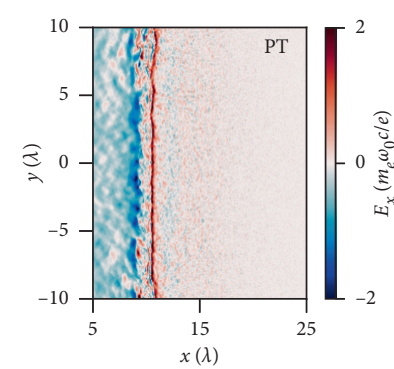

(a)

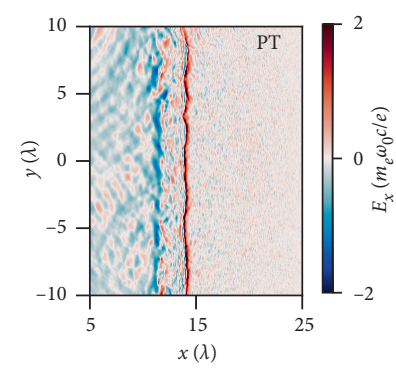

(d)

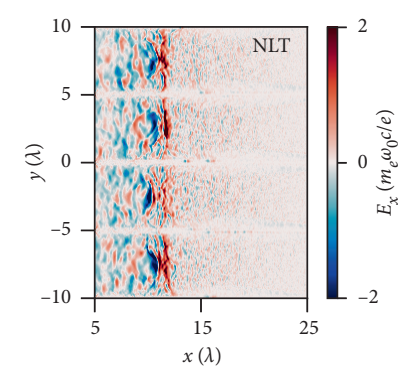

(b)

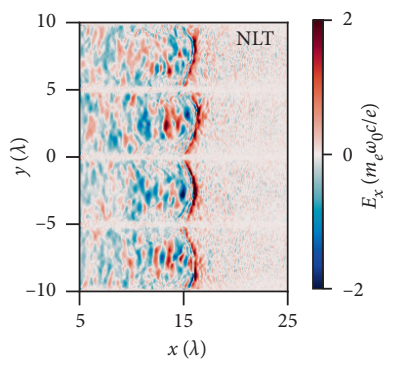

(e)

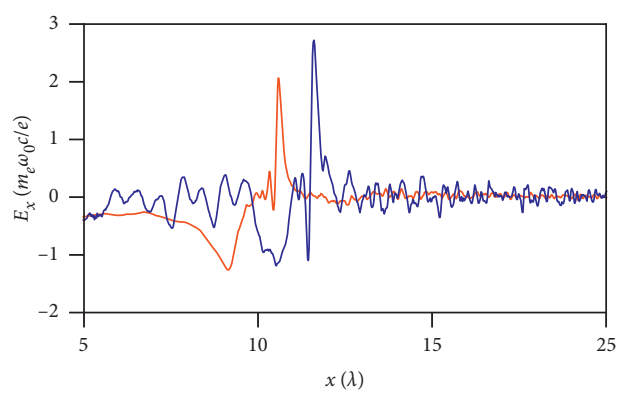

- PT

(c)

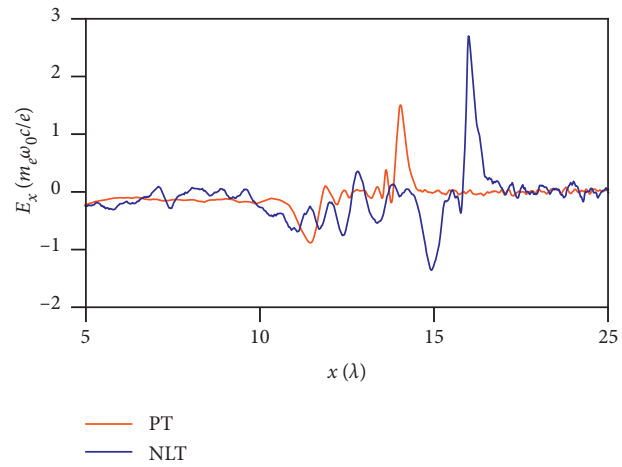

(f)

Figure 7: Plots of $E_{x}$ distribution at $t=100 T_{0}(\mathrm{a}, \mathrm{b})$ and $t=150 T_{0}(\mathrm{~d}, \mathrm{e})$ for the PT and the NLT, respectively. $E_{x}$ averaged over the transverse direction at (c) $t=100 T_{0}$ and (f) $t=150 T_{0}$.

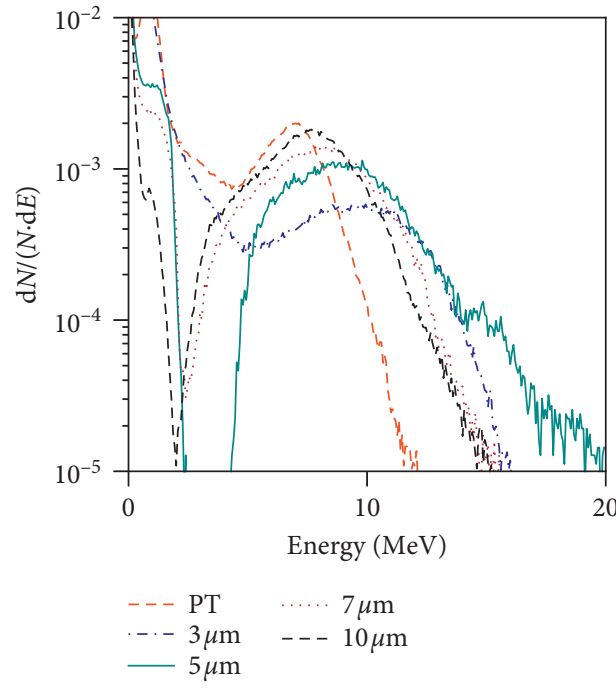

FIGURE 8: The proton energy spectrum for the PT and the NLT with different widths at $t=150 T_{0}$.

an increased ion acoustic speed, which results in a lower Mach number and a decrease in the efficiency of the CSA. Thus, the optimal gap width $d_{2}=5 \mu \mathrm{m}$ is achieved for the current NLT configuration.

\section{Summary}

In this paper, the effects of nanolayered targets on the laserdriven collisionless shock acceleration are investigated via 2D3V PIC simulations. To conclude, a stable and strong collisionless shock can be formed in the near-critical plasma embedding with a nanolayered target. More hot electrons are generated due to the interaction of the nanolayers and the incident laser. Besides, the current generated along the nanolayers induces a strong transverse magnetic field in the near-critical plasma surrounding nanolayers, which helps to inhibit the longitudinal propagation of hot electrons and accumulate them near the shock front. Compared with the planar target case, shock velocity increased from $0.079 c$ to $0.091 c$, and the electric field associated with the shock front is also reinforced. Consequently, both peak energy and cutoff energy of the accelerated protons in the upstream region are increased by as much as 30\%. Furthermore, we investigated the effect of the gap width. As the gap width decreases, the peak energy and cutoff energy increase. However, when the gap is too narrow, the quasimonoenergetic feature of CSA is impaired, and the proportion of high-energy protons declines. Our simulations provide a potential scheme for the enhancement of the CSA in experiments while careful and detailed investigations concerning the laser and target parameters for a more realistic setup should be considered in future work. 


\section{Data Availability}

The data that support the findings of this study are available from the corresponding author upon reasonable request.

\section{Conflicts of Interest}

The authors declare that they have no conflicts of interest.

\section{Acknowledgments}

This work was supported by the National Natural Science Foundation of China (Grant nos. 11875091 and 11975059), the National Key Research and Development Program of China (Grant no. 2016YFA0401100), and the Science and Technology on Plasma Physics Laboratory at CAEP (Grant no. JCKYS2019212012).

\section{References}

[1] A. Macchi, M. Borghesi, and M. Passoni, "Ion acceleration by superintense laser-plasma interaction," Reviews of Modern Physics, vol. 85, pp. 751-793, 2013.

[2] S. Bolaños, J. Béard, G. Revet et al., "Highly-collimated, highcharge and broadband $\mathrm{MeV}$ electron beams produced by magnetizing solids irradiated by high-intensity lasers," Matter and Radiation at Extremes, vol. 4, no. 4, 2019.

[3] X. T. He, H. Cai, S. Wu et al., "Physical studies of fast ignition in China," Plasma Physics and Controlled Fusion, vol. 57, Article ID 064003, 2015.

[4] X.-X. Yan, H.-B. Cai, W.-S. Zhang et al., "Anomalous mix induced by a collisionless shock wave in an inertial confinement fusion hohlraum," Nuclear Fusion, vol. 59, Article ID 106016, 2019.

[5] X. T. He, J. W. Li, Z. F. Fan et al., "A hybrid-drive nonisobaricignition scheme for inertial confinement fusion," Physics of Plasmas, vol. 23, Article ID 082706, 2016.

[6] T. Gong, L. Hao, Z. Li et al., "Recent research progress of laser plasma interactions in Shenguang laser facilities," Matter and Radiation at Extremes, vol. 4, no. 5, Article ID 055202, 2019.

[7] S. V. Bulanov, T. Z. Esirkepov, V. S. Khoroshkov, A. V. Kuznetsov, and F. Pegoraro, "Oncological hadrontherapy with laser ion accelerators," Physics Letters A, vol. 299, no. 2-3, pp. 240-247, 2002.

[8] D. Schardt, "Tumor therapy with high-energy carbon ion beams," Nuclear Physics A, vol. 787, pp. 633-641, 2007.

[9] M. Borghesi, A. Schiavi, D. H. Campbell et al., "Proton imaging detection of transient electromagnetic fields in laserplasma interactions (invited)," Review of Scientific Instruments, vol. 74, pp. 1688-1693, 2003.

[10] M. Borghesi, A. J. Mackinnon, D. H. Campbell et al., "Multi$\mathrm{MeV}$ proton source investigations in ultraintense laser-foil interactions," Physical Review Letters, vol. 92, 2004.

[11] R. A. Snavely, M. H. Key, S. P. Hatchett et al., "Intense highenergy proton beams from petawatt-laser irradiation of solids," Physical Review Letters, vol. 85, no. 14, pp. 2945-2948, 2000.

[12] S. P. Hatchett, C. G. Brown, T. E. Cowan et al., "Electron, photon, and ion beams from the relativistic interaction of Petawatt laser pulses with solid targets," Physics of Plasmas, vol. 7, no. 5, pp. 2076-2082, 2000.
[13] T. V. Liseykina, M. Borghesi, A. Macchi, and S. Tuveri, "Radiation pressure acceleration by ultraintense laser pulses," Plasma Physics and Controlled Fusion, vol. 50, 2008.

[14] B. Qiao, M. Zepf, M. Borghesi, and M. Geissler, "Stable GeV ion-beam acceleration from thin foils by circularly polarized laser pulses," Physical Review Letters, vol. 102, no. 14, 2009.

[15] T. Esirkepov, M. Borghesi, S. V. Bulanov, G. Mourou, and T. Tajima, "Highly efficient relativistic-ion generation in the laser-piston regime," Physical Review Letters, vol. 92, no. 17, 2004.

[16] L. Yin, B. J. Albright, B. M. Hegelich et al., "Monoenergetic and $\mathrm{GeV}$ ion acceleration from the laser breakout afterburner using ultrathin targets," Physics of Plasmas, vol. 14, 2007.

[17] A. Henig, D. Kiefer, K. Markey et al., "Enhanced laser-driven ion acceleration in the relativistic transparency regime," Physical Review Letters, vol. 103, 2009.

[18] T. Nakamura and K. Mima, "Magnetic-dipole vortex generation by propagation of ultraintense and ultrashort laser pulses in moderate-density plasmas," Physical Review Letters, vol. 100, no. 20, 2008.

[19] A. V. Kuznetsov, T. Z. Esirkepov, F. F. Kamenets, and S. V. Bulanov, "Efficiency of ion acceleration by a relativistically strong laser pulse in an underdense plasma," Plasma Physics Reports, vol. 27, no. 3, pp. 211-220, 2001.

[20] D. W. Forslund and J. P. Freidberg, "Theory of laminar collisionless shocks," Physical Review Letters, vol. 27, pp. 1189-1192, 1971.

[21] L. O. Silva, M. Marti, J. R. Davies et al., "Proton shock acceleration in laser-plasma interactions," Physical Review Letters, vol. 92, no. 1, 2004.

[22] J. Denavit, "Absorption of high-intensity subpicosecond lasers on solid density targets," Physical Review Letters, vol. 69, p. 3052, 1992.

[23] C. A. J. Palmer, N. P. Dover, I. Pogorelsky et al., "Monoenergetic proton beams accelerated by a radiation pressure driven shock," Physical Review Letters, vol. 106, 2011.

[24] H. Zhang, B. F. Shen, W. P. Wang et al., "Collisionless shock acceleration of high-flux quasimonoenergetic proton beams driven by circularly polarized laser pulses," Physical Review Letters, vol. 119, no. 16, 2017.

[25] F. Fiuza, A. Stockem, E. Boella et al., "Ion acceleration from laser-driven electrostatic shocks," Physics of Plasmas, vol. 20, Article ID 056304, 2013.

[26] W.-s. Zhang, H.-b. Cai, L.-1. Wei, J.-m. Tian, and S.-p. Zhu, "Enhanced ion acceleration in the ultra-intense laser driven magnetized collisionless shocks," New Journal of Physics, vol. 21, no. 4, Article ID 043026, 2019.

[27] J. M. Tian, H. B. Cai, W. S. Zhang, E. H. Zhang, B. Du, and S. P. Zhu, "Generation mechanism of $100 \mathrm{MG}$ magnetic fields in the interaction of ultra-intense laser pulse with nanostructured target," High Power Laser Science and Engineering, vol. 8, 2020.

[28] V. Kaymak, A. Pukhov, V. N. Shlyaptsev, and J. J. Rocca, "Nanoscale ultradense Z-pinch formation from laser-irradiated nanowire arrays," Physical Review Letters, vol. 117, no. 3, 2016.

[29] G. Chatterjee, P. K. Singh, S. Ahmed et al., "Macroscopic transport of mega-ampere electron currents in aligned carbon-nanotube Arrays," Physical Review Letters, vol. 108, no. 23, 2012.

[30] Z. Zhao, L. Cao, L. Cao et al., "Acceleration and guiding of fast electrons by a nanobrush target," Physics of Plasmas, vol. 17, Article ID 123108, 2010. 
[31] L. Cao, Y. Gu, Z. Zhao et al., "Control of the hot electrons produced by laser interaction with nanolayered target," Physics of Plasmas, vol. 17, Article ID 103106, 2010.

[32] Z. Samsonova, S. Höfer, V. Kaymak et al., "Relativistic interaction of long-wavelength ultrashort laser pulses with nanowires," Physical Review X, vol. 9, 2019.

[33] J. Yu, Z. Zhao, X. Jin et al., "Laser-driven proton acceleration using a conical nanobrush target," Physics of Plasmas, vol. 19, Article ID 053108, 2012.

[34] D. B. Zou, D. Y. Yu, X. R. Jiang et al., "Enhancement of target normal sheath acceleration in laser multi-channel target interaction," Physics of Plasmas, vol. 26, Article ID 123105, 2019.

[35] R. Xie, L. H. Cao, Y. Chao et al., "Improvement of laser absorption and control of particle acceleration by subwavelength nanowire target," Physics of Plasmas, vol. 27, Article ID 123108, 2020.

[36] A. Moreau, R. Hollinger, C. Calvi et al., "Enhanced electron acceleration in aligned nanowire arrays irradiated at highly relativistic intensities," Plasma Physics and Controlled Fusion, vol. 62, Article ID 014013, 2019.

[37] M. Dozières, "Optimization of laser-nanowire target interaction to increase the proton acceleration efficiency," Plasma Physics and Controlled Fusion, vol. 61, 2019.

[38] S. Kahaly, S. K. Yadav, W. M. Wang et al., "Near-complete absorption of intense, ultrashort laser light by sub- $\lambda$ gratings," Physical Review Letters, vol. 101, no. 14, 2008.

[39] R. Hollinger, C. Bargsten, V. N. Shlyaptsev et al., "Efficient picosecond X-ray pulse generation from plasmas in the radiation dominated regime," Optica, vol. 4, 2017.

[40] D. Khaghani, M. Lobet, B. Borm et al., "Enhancing laserdriven proton acceleration by using micro-pillar arrays at high drive energy," Scientific Reports, vol. 7, no. 1, 2017.

[41] G. Cristoforetti, P. Londrillo, P. K. Singh et al., "Transition from Coherent to Stochastic electron heating in ultrashort relativistic laser interaction with structured targets," Scientific Reports, vol. 7, no. 1, p. 1479, 2017.

[42] D. S. Black, K. J. Leedle, Y. Miao, U. Niedermayer, R. L. Byer, and O. Solgaard, "Laser-driven electron lensing in silicon microstructures," Physical Review Letters, vol. 122, no. 10, 2019.

[43] I. Prencipe, A. Sgattoni, D. Dellasega et al., "Development of foam-based layered targets for laser-driven ion beam production," Plasma Physics and Controlled Fusion, vol. 58, 2016.

[44] T. D. Arber, K. Bennett, C. S. Brady et al., "Contemporary particle-in-cell approach to laser-plasma modelling," Plasma Physics and Controlled Fusion, vol. 57, 2015.

[45] L. Cao, Y. Gu, Z. Zhao et al., "Enhanced absorption of intense short-pulse laser light by subwavelength nanolayered target," Physics of Plasmas, vol. 17, Article ID 043103, 2010.

[46] H. He, B. Qiao, X. F. Shen et al., "High-flux high-energy ion beam production from stable collisionless shock acceleration by intense petawatt-picosecond laser pulses," New Journal of Physics, vol. 21, no. 3, Article ID 033035, 2019.

[47] A. Macchi, F. Cattani, T. V. Liseykina, and F. Cornolti, "Laser acceleration of ion bunches at the front surface of overdense plasmas," Physical Review Letters, vol. 94, no. 16, 2005.

[48] G. Sarri, M. E. Dieckmann, I. Kourakis, and M. Borghesi, "Generation of a purely electrostatic collisionless shock during the expansion of a dense plasma through a rarefied medium," Physical Review Letters, vol. 107, no. 2, 2011. 